\title{
The Yin and Yang actions of North American ginseng root in modulating the immune function of macrophages
}

\author{
Chike Godwin Azike ${ }^{1,2}$, Paul Abrahams Charpentier ${ }^{1,3}$, Jirui Hou ${ }^{1,2}$, Hua Pei ${ }^{1,2}$ and Edmund Man King Lui ${ }^{1,2^{*}}$
}

\begin{abstract}
Background: Immuno-modulatory effects of ginseng, including both immuno-stimulatory and immunosuppressive effects, have been widely reported. This study aims to determine whether the paradoxical immunomodulatory effect is related to unique phytochemical profiles of different North American (NA) ginseng, namely aqueous (AQ) and alcoholic (ALC) extracts.
\end{abstract}

Methods: $A Q$ and ALC extracts were prepared and their immuno-bioactivity were studied in vitro in murine macrophages (Raw 264.7) through measuring the direct stimulatory production of pro-inflammatory mediator and cytokines as well as the suppression of lipopolysaccharide (LPS)-stimulatory response by the two extracts. Gel permeation chromatography was used to fractionate and isolate phytochemicals for characterization of ginseng extracts.

Results: AQ extract up-regulated the production of nitric oxide (NO), tumour necrosis factor- $\alpha$ (TNF- $\alpha$ ) and interleukin-6 (IL-6) while ALC extract did not. ALC extract but not AQ extract suppressed LPS-induced macrophage $\mathrm{NO}$ and TNF- $\alpha$ production. These immuno-stimulatory and suppressive effects were exhibited at similar extract concentrations. Moreover, the macrophage-stimulating activity of the AQ extract was inhibited in the presence of ALC extract. Fractionation of $\mathrm{AQ}$ extract revealed the presence of two major peaks at $230 \mathrm{~nm}$ with average molecular weights of 73,000 and 37,000 Da. The first fraction had similar elution volume as the crude polysaccharide (PS) fraction isolated from the AQ extract, and it was the only bioactive species. Parallel fractionation study of ALC extract yielded similar elution profiles; however, both sub-fractions were devoid of PS. Fraction I of the ALC extract suppressed LPS-induced NO production dose-dependently.

Conclusion: ALC extract of NA ginseng, which was devoid of PS, was immuno-inhibitory whereas the AQ extract, which contained PS, was immuno-stimulatory. These extract-related anti-inflammatory and pro-inflammatory effects may be considered as the Yin and Yang actions of ginseng.

\section{Background}

Ginseng is a perennial herb of the Araliaceae family. Asian ginseng (Panax ginseng C.A. Meyer, Renshen) and NA ginseng (Panax quinquefolius L., Xiyangshen) are the most commonly used ginseng species. While Asian ginseng has been used for thousands of years as tonic to improve overall health, restore the body to balance, help the body to heal itself and reduce stress [1], the medicinal use of NA ginseng traces back about 400 years ago.

\footnotetext{
* Correspondence: Ed.lui@schulich.uwo.ca

'Ontario Ginseng Innovation and Research Consortium, the University of

Western Ontario, London, Ontario, N6A 5C1, Canada

Full list of author information is available at the end of the article
}

Canada is currently the largest producer of NA ginseng [1-3]. Recognized by the Canadian regulatory agency as a natural health product for use as an adaptogen (biological response modifier) [4], NA ginseng is a multiaction herb with a wide range of pharmacological effects on the central nervous system, cardiovascular system and endocrine secretion, reproductive and immune function [5].

Ginseng has influences on both the innate and adaptive immunity. Macrophage-mediated innate immunity is the first line of defence against microbial pathogens and influences the subsequent adaptive immune response. Macrophages kill pathogens and cancer cells
C Biomed Central

(C) 2011 Azike et al; licensee BioMed Central Ltd. This is an Open Access article distributed under the terms of the Creative Commons Attribution License (http://creativecommons.org/licenses/by/2.0), which permits unrestricted use, distribution, and reproduction in any medium, provided the original work is properly cited. 
directly via phagocytosis and indirectly via the production of various pro-inflammatory mediators (e.g. NO) and cytokines (e.g. TNF- $\alpha$ ) [6]. However, over production of pro-inflammatory mediators [7] may result in inflammatory diseases and/or tissue injury which are then managed by immune-suppressive agents. Modulation of macrophage function, e.g. up-regulation of inflammatory mediator production in vitro or suppression of its stimulation by LPS has been used as an experimental tool to evaluate immuno-stimulatory and anti-inflammatory potency of herbal products respectively [8].

Ginseng contains bioactive compounds such as ginsenosides, which are steroidal saponins containing different sugar moieties and possessing different lipidsolubility [5] and polysaccharides (PS) consisting of complex chain of monosaccharides rich in L-arabinose, D-galactose, L-rhamnose, D-galacturonic acid, D-glucuronic acid and D-galactosyl residue $[9,10]$. Choice of solvents influences the bioactive components in the extracts. This factor is often overlooked by many investigators who focus mainly on biological activities.

Inconsistent immuno-modulatory effects of ginseng have been reported, including both immuno-stimulatory and immuno-suppressive effects [11-22]. The basis for the apparent paradoxical immuno-modulatory effects is unclear but may be attributed to different experimental conditions, e.g. choice of extraction solvents.

The objectives of this study are (1) to characterize the apparent paradoxical effects of ginseng by examining the immuno-modulatory effect of AQ and ALC extracts prepared from 4-year-old Ontario grown NA ginseng roots in RAW 264.7 murine macrophage cell line and (2) to explore the characteristics of the immuno-modulatory bioactive substances of ginseng.

\section{Methods}

\section{Ginseng and its extracts}

Four-year-old NA ginseng roots collected in 2007 from five different farms in Ontario, Canada were provided by the Ontario Ginseng Growers Association. Ginseng extracts from each farm were prepared individually and combined to produce composite extracts which were used for phytochemical and pharmacological studies.

\section{Materials}

RAW 264.7 (ATCC TIB 67) murine macrophage cell lines were provided by Dr Jeff Dixon (Department of Physiology and Pharmacology, University of Western Ontario, Canada). Sephadex G75 was purchased from GE healthcare bio-sciences AB (Sweden). Cell culture medium and reagents were purchased from Gibco laboratories (USA). BD OptEIA ELISA kits tumour necrosis factor- $\alpha$ and interleukin-6 (BD Biosciences,
USA). LPS from Escherichia coli and Griess reagent were purchased from Sigma-Aldrich (USA).

\section{Preparation of the AQ, ALC and crude PS ginseng extracts}

Dried ginseng root samples were shipped to Naturex (USA) for extraction. Samples were ground between $1 / 4$ and $1 / 2$ inch and used to produce the AQ or ALC extract. Briefly, $4 \mathrm{~kg}$ ground ginseng roots were soaked three times during five hours in $16 \mathrm{~L}$ of water or etha$\mathrm{nol} /$ water $(75 / 25, \mathrm{v} / \mathrm{v})$ solution at $40^{\circ} \mathrm{C}$. After extraction, the solution was filtered at room temperature. The excess solvent was then removed by a rotary evaporator under vacuum at $45^{\circ} \mathrm{C}$. The three pools were combined and concentrated again until the total solids on a dry basis were around $60 \%$. These concentrates were lyophilized with a freeze dryer (Labconco, USA) at $-50^{\circ} \mathrm{C}$ under reduced pressure to produce AQ or ALC ginseng extract in powder form. Yield of the powder extracts from the concentrates was about $66 \%$. The yields of the final extract (mean \pm standard deviation of $\%$ extractive) from the initial ground root were $41.74 \pm 4.92$ and $35.30 \pm 5.01$ for the AQ and ALC extracts respectively.

A solution of AQ extract in distilled water $(10 \mathrm{~g} / 10$ $\mathrm{mL}$ ) was prepared, and the crude PS was precipitated by the addition of four volumes of $95 \%$ ethanol. The PS fraction was collected by centrifugation at $350 \times g$ (Beckman Model TJ-6, USA) for 10 minutes and lyophilized to produce the crude PS extract.

\section{Chromatography of ginseng extracts \\ High performance liquid chromatography (HPLC) analysis for ginsenoside determination}

HPLC analysis on the composition of ginsenosides in AQ and ALC extracts (100 mg/ml methanol) was performed with a Waters 1525 HPLC System with a binary pump and UV detector. A reversed-phase Inspire C18 column $(100 \mathrm{~mm} \times 4.6 \mathrm{~mm}$, i.d. $5 \mu \mathrm{m})$ purchased from Dikma Technologies (USA) was used for all chromatographic separations. Gradient elution consisted of [A] water and [B] acetonitrile at a flow of $1.3 \mathrm{~mL} / \mathrm{min}$ as follows: $0 \mathrm{~min}, 80-20 \%$; 0-60 $\mathrm{min}, 58-42 \%$; $60-70 \mathrm{~min}$, $10-90 \%$; 70-80 $\mathrm{min}, 80-20 \%$. Absorbance of the eluates was monitored at $203 \mathrm{~nm}$.

\section{Sephadex G-75 chromatography}

Five hundred milligrams (500 mg) of AQ or ALC ginseng extract was dissolved in $5 \mathrm{~mL}$ distilled water and then fractionated by loading to a calibrated Sephadex G-75 column $(47 \times 2.5 \mathrm{~cm})$ equilibrated and eluted with distilled water mobile phase at $4^{\circ} \mathrm{C}$ with a flow rate of $1 \mathrm{~mL} / \mathrm{min}$. Absorbance of the eluates was monitored at $230 \mathrm{~nm}$. Fractions $(5 \mathrm{~mL})$ were collected and four major fractions (I-IV) were collected and lyophilized to produce four sub-fractions (I-IV) for the study of bioactivity distribution. 


\section{Size exclusion chromatography for PS analysis}

Size exclusion chromatography of AQ, ALC and PS ginseng extract was carried out at $40^{\circ} \mathrm{C}$ with an AquaGel PAA-200 Series column $(8 \times 300 \mathrm{~mm}$, PolyAnalytik, USA) connected to a Viscotek (Varian Instruments, USA) gel permeation chromatography system with Omnisec software (version 4.5, Viscotek, USA) for data acquisition. Solutions of AQ, ALC and PS extract (5 $\mathrm{mg} / \mathrm{mL}$ ) were filtered with $0.2 \mu \mathrm{m}$ nylon filter and used for analysis. Each sample $(100 \mu \mathrm{l})$ was injected and eluted with $0.05 \mathrm{M}$ sodium nitrate $\left(\mathrm{NaNO}_{3}\right)$ mobile phase at a flow rate of $1 \mathrm{~mL} / \mathrm{min}$ and monitored using a multiple detectors system for light scattering, refractive index and viscosity. Pullulan polysaccharide reference standard was analyzed as a positive control.

\section{Cell culture}

Mouse macrophage cell line RAW 264.7 was cultured in Dulbeccos Modified Eagle's Medium supplemented with 10\% Fetal Bovine Serum, 25 mM HEPES, 2 mM Glutamine, $100 \mathrm{IU} / \mathrm{ml}$ penicillin and $100 \mu \mathrm{g} / \mathrm{ml}$ streptomycin. Cells were seeded in 96-well tissue culture plates at a density of $1.5 \times 10^{5}$ cells per well and maintained at $37^{\circ}$ $\mathrm{C}$ in a humidified incubator with $5 \% \mathrm{CO}_{2}$ and weekly passage and used for experiments at $60-80 \%$ confluency.

\section{Cell treatment}

\section{Immuno-stimulatory effect}

Experiment to evaluate dose-related stimulation of inflammatory mediators profile in vitro was carried out by treating and incubating macrophages $\left(1.5 \times 10^{5}\right.$ cells/ well) with $0,20,50$ and $200 \mu \mathrm{g} / \mathrm{ml}$ of ginseng extracts or $1 \mu \mathrm{g} / \mathrm{mL}$ of LPS (positive control) for 24 hours. The end-points were the 24 hours-production of NO, TNF- $\alpha$ and IL-6 inflammatory mediators.

\section{Immuno-suppression of LPS-induced effect}

To examine the direct inhibitory effect of ginseng extracts on LPS-stimulated immune function, we pretreated the macrophages with $0,10,50,100$ or $200 \mu \mathrm{g} /$ $\mathrm{ml}$ of ginseng extracts two hours prior to the addition of $1 \mu \mathrm{g} / \mathrm{mL}$ of LPS. The 24-hour cytokine production induced by LPS was determined by measuring NO, TNF- $\alpha$ and IL- 6 levels in the culture medium.

\section{Suppression of $A Q$ extract-induced macrophage NO} stimulation by $A L C$ extract

Production of NO by $1.5 \times 10^{5}$ macrophages/well in a 96 well-plate induced by 0,50 and $200 \mu \mathrm{g} / \mathrm{ml}$ of AQ ginseng extract was determined 24 hours after the presence and absence of $200 \mu \mathrm{g} / \mathrm{ml}$ ALC ginseng extract.

\section{Quantification of NO, TNF- $\alpha$ and IL-6}

TNF- $\alpha$ and IL- 6 concentrations in supernatants from cultured cells were analyzed with ELISA. Samples were evaluated with mouse cytokine-specific BD OptEIA ELISA kits (BD Biosciences, USA) according to the manufacturer's protocol. NO production was analyzed as accumulation of nitrite in the culture medium. Nitrite in culture supernatants was determined with Griess reagent (Sigma-Aldrich, USA). Briefly, $50 \mu \mathrm{L}$ of culture supernatant from each sample were transferred to wells of a 96-well U-bottom microtiter plate, $50 \mu \mathrm{L}$ Griess reagent (containing $0.5 \%$ sulfanilic acid, $0,002 \% \mathrm{~N}-1$ naphtyl-ethylenediamine dihydrochloride and $14 \%$ glacial acetic acid) was then added. The absorbance at 550 $\mathrm{nm}$ wavelength was measured using Multiskan Spectrum microplate reader (Thermo Fisher Scientific, Finland) with SkanIt software (version 2.4.2, Thermo Fisher Scientific, Finland). Sample nitrite concentrations were estimated from a sodium nitrite standard calibration curve.

\section{Statistical analysis}

Each cell culture experiment was performed at least three separate times. All statistical analyses were performed with GraphPad prism 4.0a Software (GraphPad Software Inc., USA). Data were presented as the mean \pm standard deviation (SD) of triplicates from three independent experiments. Data sets with multiple comparisons were evaluated by one-way analysis of variance (ANOVA) with Dunnett's post-hoc test. $P<0.001$ was considered to be statistically significant.

\section{Results}

Phytochemical characteristics of the AQ and ALC ginseng extracts

HPLC analysis of the AQ and ALC ginseng extracts showed significant differences in the total ginsenoside $\left(R b_{1}, R e, R c, R d, R g_{1}\right.$ and $\left.R b_{2}\right)$ content and profiles. ALC extract contained over twice as much amount of total ginsenosides as the AQ extract, namely $28.25 \%$ vs. $13.87 \%$ dry weight of extract. $R b_{1}$ and Re were the two most predominant ginsenosides in both extracts but the $\mathrm{Rb}_{1} /$ Re ratio was higher in the ALC extract, namely 1.8 vs. 1.1. No detectable levels of $\mathrm{Rh}_{1}$ were measured.

\section{Immuno-stimulatory effect of the $\mathrm{AQ}$ and $\mathrm{ALC}$ ginseng} extracts in macrophages in vitro

Evaluation of the immuno-stimulatory effect of the ginseng extracts on RAW 264.7 murine macrophages revealed that exposure to $20-200 \mu \mathrm{g} / \mathrm{mL}$ of AQ extract significantly up-regulated macrophage production of NO, TNF- $\alpha$ and IL- 6 compared to untreated control in a concentration-dependent manner (Figure 1). The responses to $200 \mu \mathrm{g} / \mathrm{mL}$ of AQ extract in NO and TNF$\alpha$ production were similar to the maximum stimulatory response induced by $1 \mu \mathrm{g} / \mathrm{mL}$ of LPS. Moreover, the magnitude of maximum stimulatory response pertaining to NO and TNF- $\alpha$ (as a \% of the positive control) was much greater that of IL-6. By contrast, the ALC extract had no apparent immuno-stimulatory effect (Figure 1). 


\section{Aqueous extract}

A

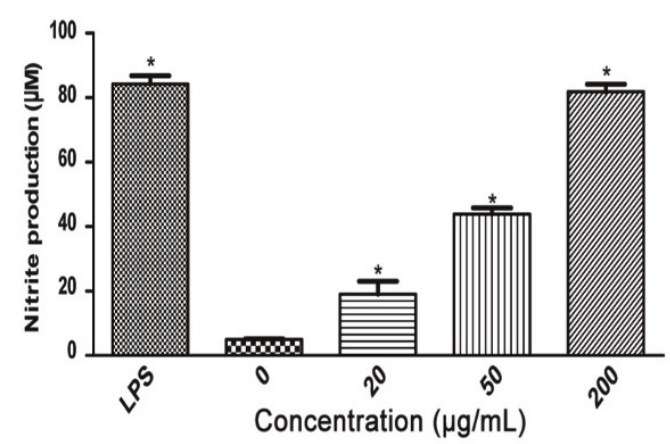

B

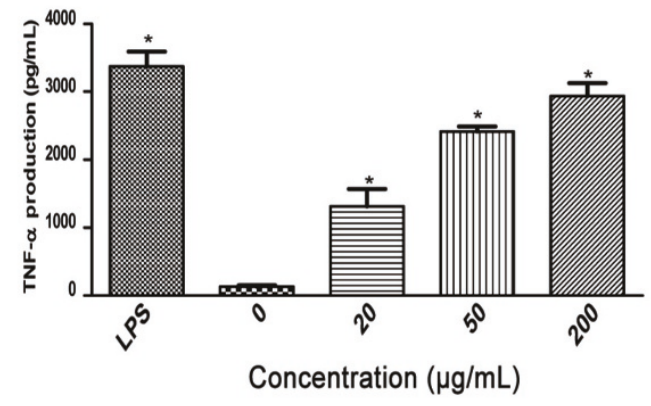

C

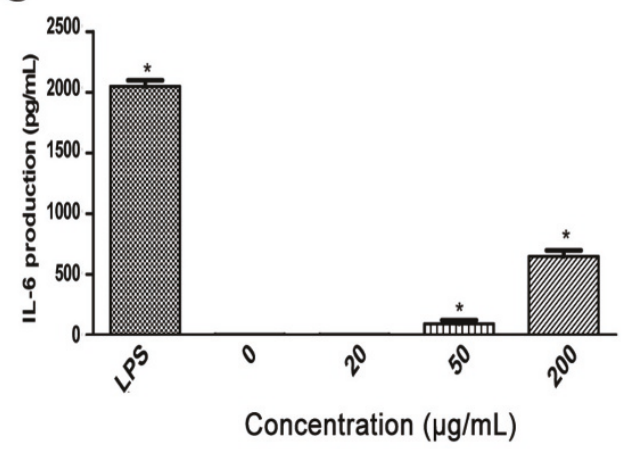

Alcoholic extract
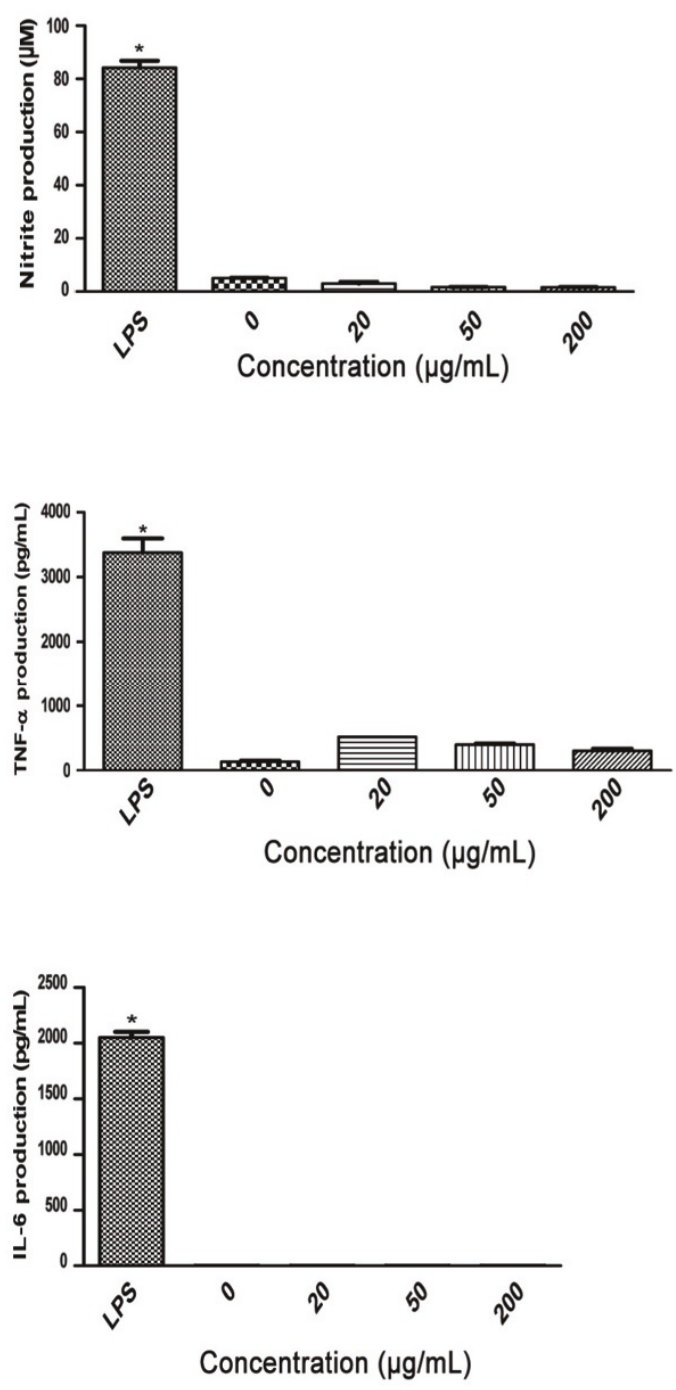

Figure 1 Immuno-stimulatory effects of the AQ and ALC ginseng extracts on 24 hours macrophage production of (A) NO, (B) TNF- $\alpha$

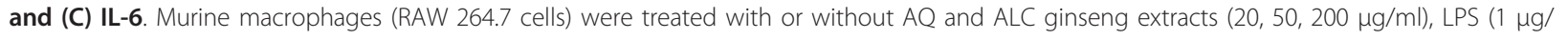
$\mathrm{ml}$ ) for 24 hours and the culture supernatants were analysed for NO and TNF- $\alpha /$ /L-6 by Griess reaction assay and ELISA respectively. Three independent experiments were performed and the data were shown as mean $\pm \mathrm{SD}$. Datasets were evaluated by ANOVA. ${ }^{*}$ Values $P<0.001$ compared to the untreated (vehicle) control were statistically significant.

Effect of the AQ and ALC ginseng extracts on LPSstimulated production of NO and TNF- $\alpha$ in macrophages in vitro

Figure 2 showed the influence of ginseng extract treatment on LPS-stimulated NO and TNF- $\alpha$ production in macrophages. LPS stimulated 24-hour production of NO markedly, which was significantly suppressed in the presence of $20-200 \mu \mathrm{g} / \mathrm{ml}$ of the ALC extract in a dosedependent manner (Figure 2). This inhibitory effect appeared to be extract-specific as the AQ extract was marginally effective and only at high concentrations (Figure 2). Figure 2 also showed that the influence of ginseng was cytokine-specific, i.e. the magnitude of inhibition by ALC extract was much smaller with respect to 


\section{Aqueous extract}

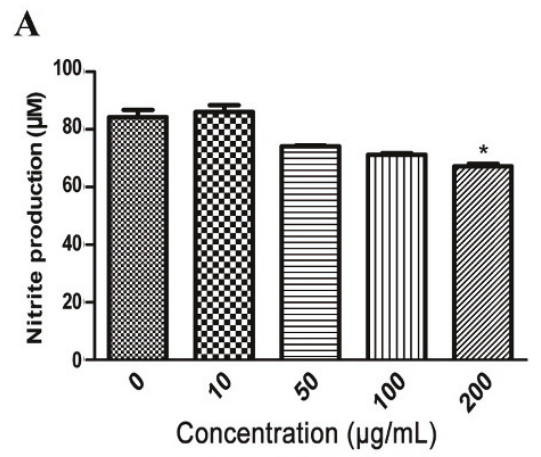

B

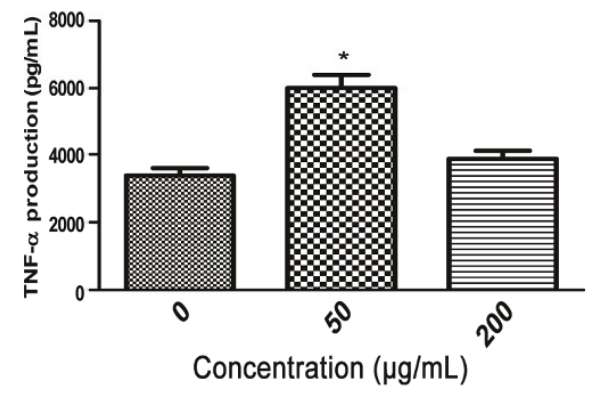

Alcoholic extract
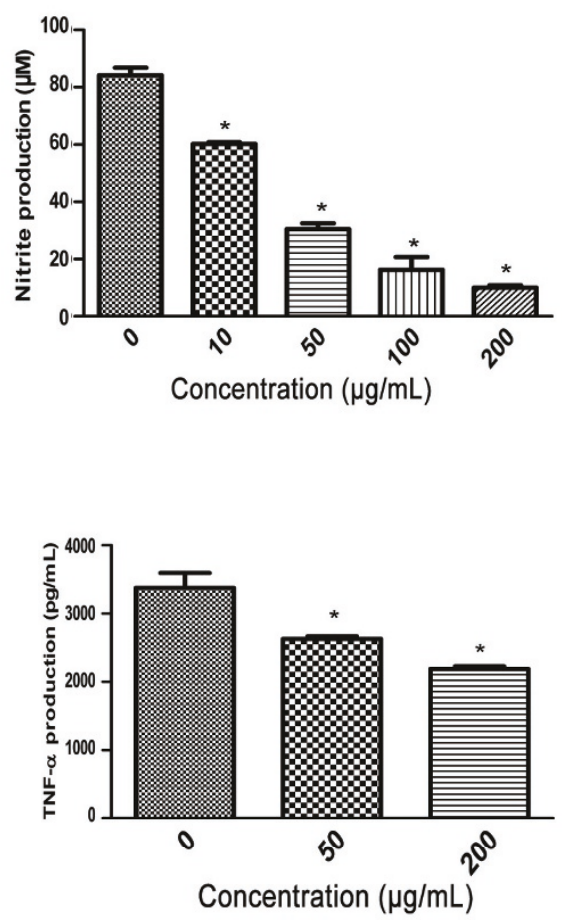

Figure 2 Effect of the AQ and ALC ginseng extracts on LPS-stimulated 24 hours macrophage production of (A) NO and (B) TNF- $\alpha$. Murine macrophages (RAW 264.7 cells) were pre-treated with without the AQ and ALC ginseng extracts (50, $200 \mu \mathrm{g} / \mathrm{ml}$ ), for two hours after which LPS $1 \mu \mathrm{g} / \mathrm{ml}$ was added; and 24 hours later the NO and TNF- $\alpha$ contents of the culture supernatants were determined by Griess reaction assay and ELISA, respectively. Three independent experiments were performed and the data were shown as mean \pm SD. Datasets were evaluated by ANOVA. * Values $P<0.001$ compared to the LPS positive control were statistically significant.

TNF- $\alpha$ production. Moreover, the AQ extract had either no inhibitory effect at high concentration or additive effect at low concentration.

\section{Suppression of the $A Q$ ginseng extract-induced immuno- stimulation by the ALC ginseng extract}

To further study the apparent extract-specific paradoxical immuno-modulatory effects of ginseng, we carried out an experiment to determine whether the immunostimulation induced by the AQ extract could be suppressed by concurrent treatment with the ALC extract. The dose-related up-regulation of $\mathrm{NO}$ production in macrophages by the AQ extract was reduced by $50-65 \%$ with exposure to equivalent concentrations of the ALC extract (Figure 3).

\section{Immuno-stimulatory and immuno-suppressive} components of the $A Q$ and ALC ginseng extracts

To further study the apparent extract-specific paradoxical immuno-modulatory effects of ginseng, we examined the extract-specific bioactive compounds that mediated these effects. Gel filtration of the AQ extract on a Sephadex G75 column resulted in the appearance of two major peaks (Fractions I and III) based on the absorbance at $230 \mathrm{~nm}$ (Figure 4A). The estimated average molecular weights of Fractions I and III were about 73,000 and 37,000 Da respectively; and their yield accounted for $28 \%$ and $40 \%$ by dry weight of the AQ extract respectively. Since PS of ginseng possesses an immuno-stimulatory effect $[9,10,13,23]$, the crude PS fraction was isolated from the AQ extract by alcohol (40\%) precipitation (with a yield of $10 \%$ by weight) and was subjected to similar chromatographic procedure for comparison. As shown in Figures $4 \mathrm{~A}$ and $4 \mathrm{C}$, the major PS peak had a similar elution volume as Fraction I of the AQ extract.

Figure 5 showed the data concerning the stimulation of cytokine production in macrophages by these fractions. Stimulatory activity of Cold-Fx, a commercial natural health product with well established immunostimulatory activity $[9,10]$, was included as a reference. The immuno-stimulatory activity with respect to NO and TNF- $\alpha$ production was associated only with 


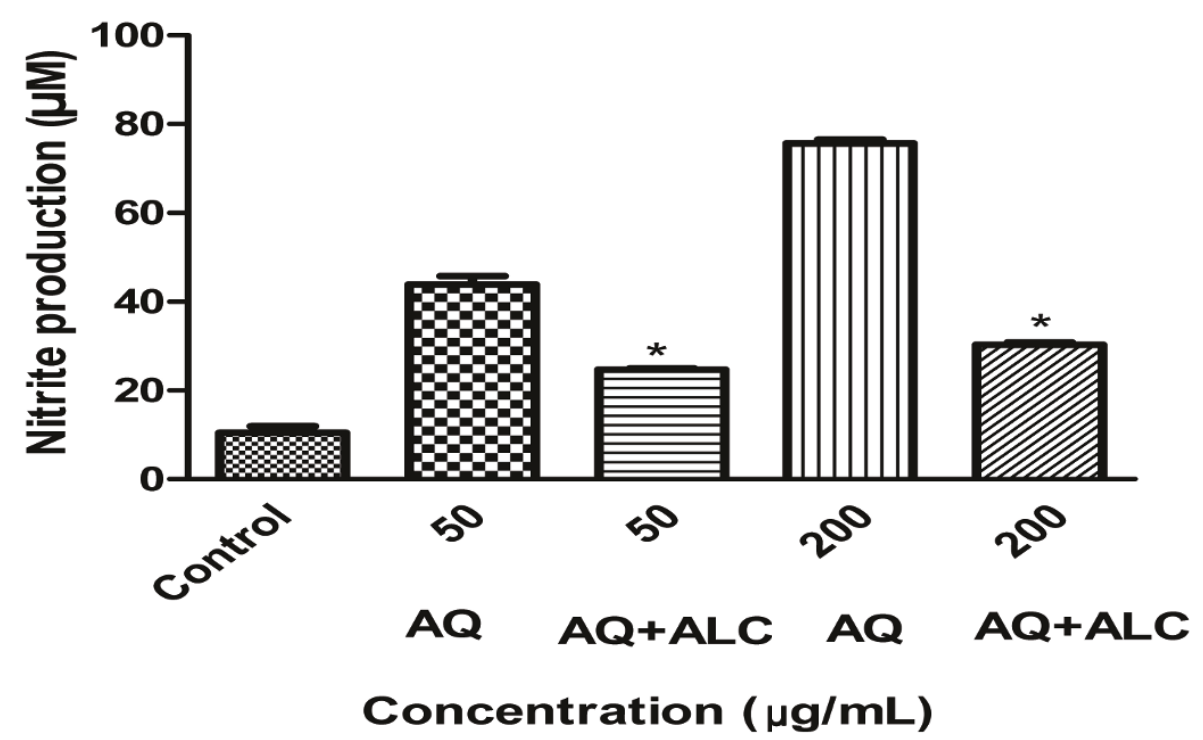

Figure 3 ALC ginseng extract suppressed up-regulation of macrophage NO production by the AQ ginseng extract. Murine macrophages (RAW 264.7 cells) were pre-treated with or without the $200 \mu \mathrm{g} / \mathrm{ml}$ ALC ginseng extracts $(50,200 \mu \mathrm{g} / \mathrm{ml})$, for two hours after which the AQ ginseng extract $(50,200 \mu \mathrm{g} / \mathrm{ml})$ was added, and the NO contents of the culture supernatants were determined by Griess reaction assay 24 hours later. Three independent experiments were performed and the data were shown as mean \pm SD. Datasets were evaluated by ANOVA. ${ }^{*}$ Values $P$ $<0.001$ were statistically significant.

macromolecules of Fraction I but not Fraction III and the potency of the former was similar to the PS extract and was better than Cold-Fx. Fraction I was less active than the PS extract in terms of IL-6 production. Since PS and Fraction I corresponded to $10 \%$ and $28 \%$ of the AQ extract by weight, it appeared that these isolated chemical constituents could only account for part of the observed immuno-stimulatory activities of the AQ extract on the basis of the difference in their immunostimulatory potency.

Fractions I (10\%) and III (64\%) obtained from Sephadex chromatographic profile of the ALC extract contained no immuno-stimulatory activity (data not shown). Fraction I was not affected by treatment with $40 \%$ ethanol (data not shown). This observation was consistent with the lack of PS in the ALC extract. Figure 6 indicated that Fraction I of ALC extract was particularly more active than Fraction III: causing significant and dose-dependent reduction in 24-hour NO production by macrophages induced by $1 \mu \mathrm{g} / \mathrm{mL}$ LPS. Moreover, treatment with $200 \mu \mathrm{g} / \mathrm{ml}$ of $\mathrm{Rb}_{1}$ and $\mathrm{Rg}_{1}$ did not have significant effects on LPS-induced 24-hour NO production in macrophages (data not shown).

In view of the similarity in the Sephadex G-75 profile of the AQ and ALC extracts, we used more specific chromatographic technique to differentiate the macromolecular constituents from the two extracts. Light scattering data in Figure 7 showed the presence of PS in Peak I of AQ extract on the basis of its similarity to the polysaccharide reference and the crude PS fraction isolated from ginseng. By contrast, Peak I of the ALC extract contained no detectable PS.

\section{Discussion}

The present study delineated the paradoxical immunomodulatory effect of ginseng and provided a basis for explaining the apparently contradictory reporting in the literature. The observed extract-specific immuno-stimulatory and immuno-suppressive effects were described independently by a number of investigators who examined the activity of the aqueous [11-15] or alcoholic [16-21] extracts of ginseng. Moreover, there was a pattern of association of immuno-stimulation and immunosuppressive activities with aqueous [11-15] and alcoholic [16-21] extracts respectively, with the exception of a study showing an aqueous extract to possess immunosuppressive effect [22]. In light of the observed paradoxical effects and similarity in the yield (\% extractive of 41.4 and 35.3) and potency of the AQ and ALC extracts (Figure 1 and 2), we consider the extract-specific inhibitory and stimulatory effect on macrophage function reported in the present study as the Yin and Yang actions of ginseng. This concept was considered as an extension of the Yin and Yang actions of ginseng proposed by other investigators on angiogenesis [24] and cancer cell proliferation $[25,26]$.

Findings on the macrophage-stimulating effect of NA ginseng (Figure 1) have provided new information on 


\section{A}

North American ginseng aqueous extract

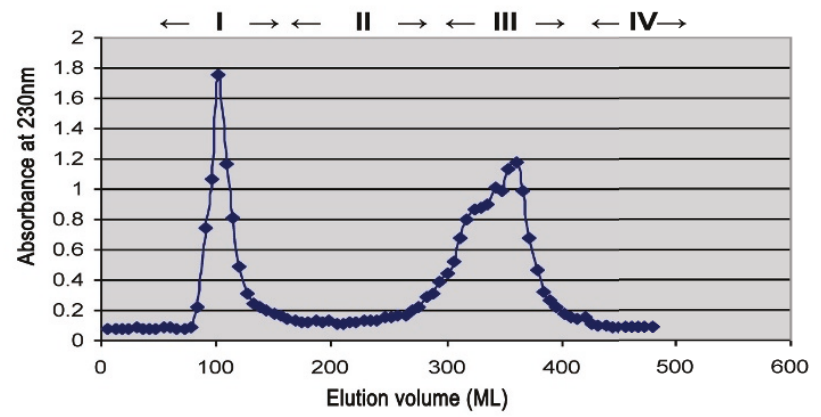

B

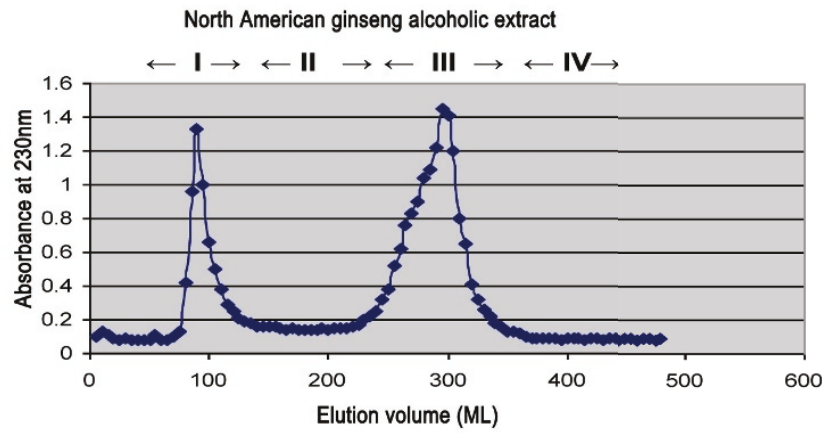

C

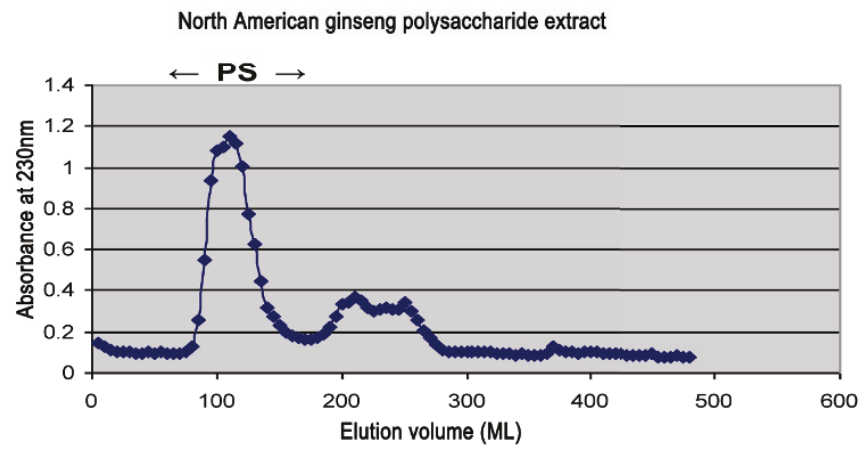

Figure 4 Sephadex G-75 $(47 \times 2.5 \mathrm{~cm})$ chromatographic fractionation of the (A) AQ, (B) ALC and (C) PS extracts of ginseng. Column was loaded with $500 \mathrm{mg}$ of extract, and then eluted with distilled water at flow rate of $1 \mathrm{~mL} / \mathrm{min}$. The $y$-axis is the absorbance at $230 \mathrm{~nm}$ while the $x$-axis represents the elution volume $(\mathrm{mL})$.

the immuno-stimulatory property of ginseng in term of its cytokine specificity and dose dependency, which also reflected on the specific pharmacological basis of its biological activity. In a separate study we have also demonstrated that the same AQ extract stimulated inflammatory cytokines (IL-1 $\beta$, IL-6, TNF- $\alpha$ ) as well as IL-10 response by human peripheral blood mononuclear cells (PBMC) [27]. Moreover, the changes reported above were not due to LPS contamination of the extracts as documented by Limulus test and direct LPS assay. The potency of the AQ extract was significant in that its stimulatory activities per unit weight is either similar or better than that of Cold-Fx, a licensed Canadian Natural Health Product enriched in polysaccharides 


\section{NO production}

A

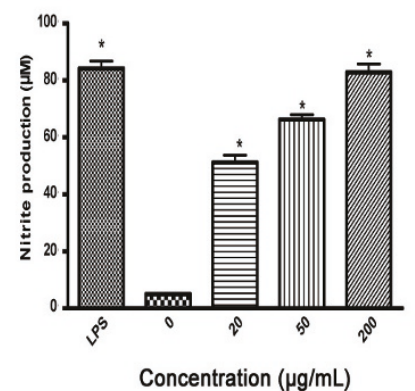

B

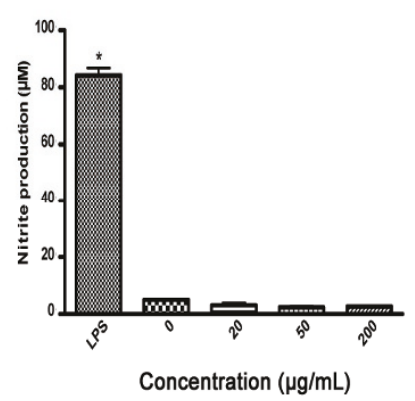

C

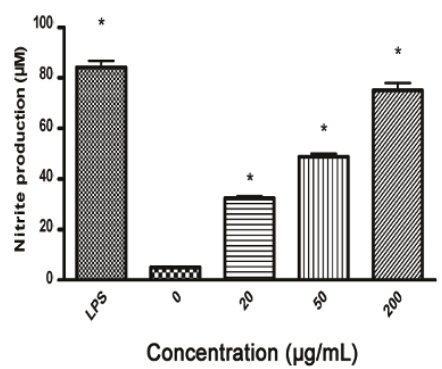

D

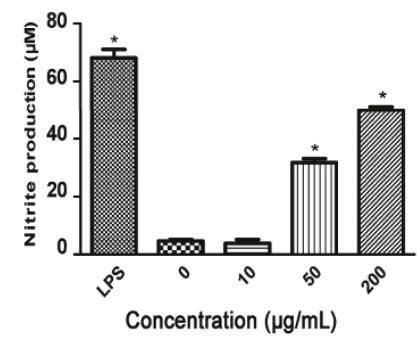

TNF- $\alpha$ production

\section{IL-6 production}
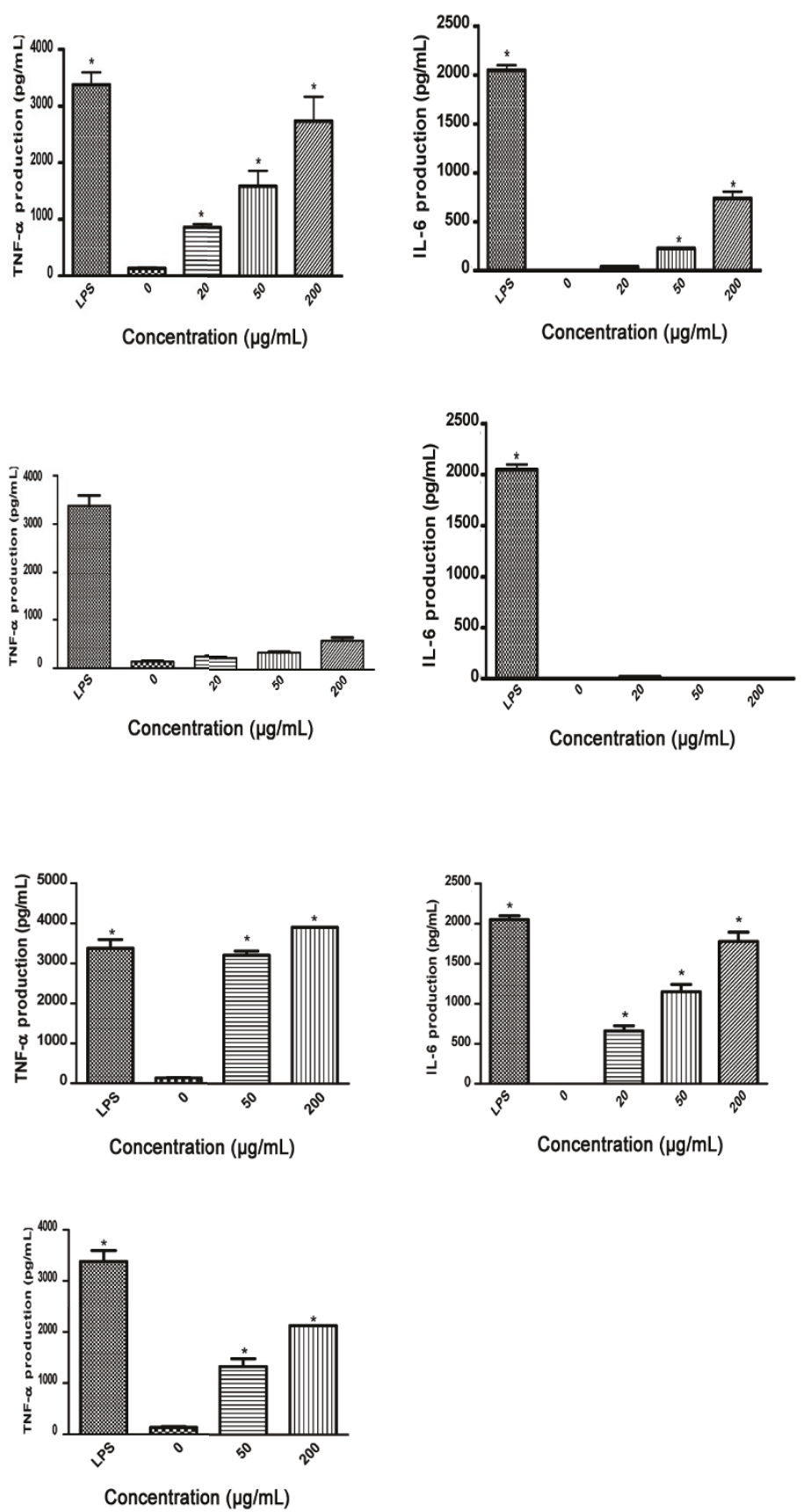

Figure 5 Immuno-stimulatory effect of Fraction I and III of the AQ, PS extracts of ginseng and Cold-Fx. Murine macrophages (RAW 264.7 cells) were treated with Fraction I and III of AQ ginseng extract, PS extract of ginseng and Cold-Fx. (0, 20, 50, $200 \mu \mathrm{g} / \mathrm{ml}), \mathrm{LPS}(1 \mu \mathrm{gg} / \mathrm{ml})$ for 24 hours and the NO, TNF- $\alpha$ and IL-6 contents of the culture supernatants were determined. Three independent experiments were performed and the data were shown as mean \pm SD. Datasets were evaluated by ANOVA. ${ }^{*}$ Values $P<0.001$ compared to the untreated (vehicle) control were statistically significant. 

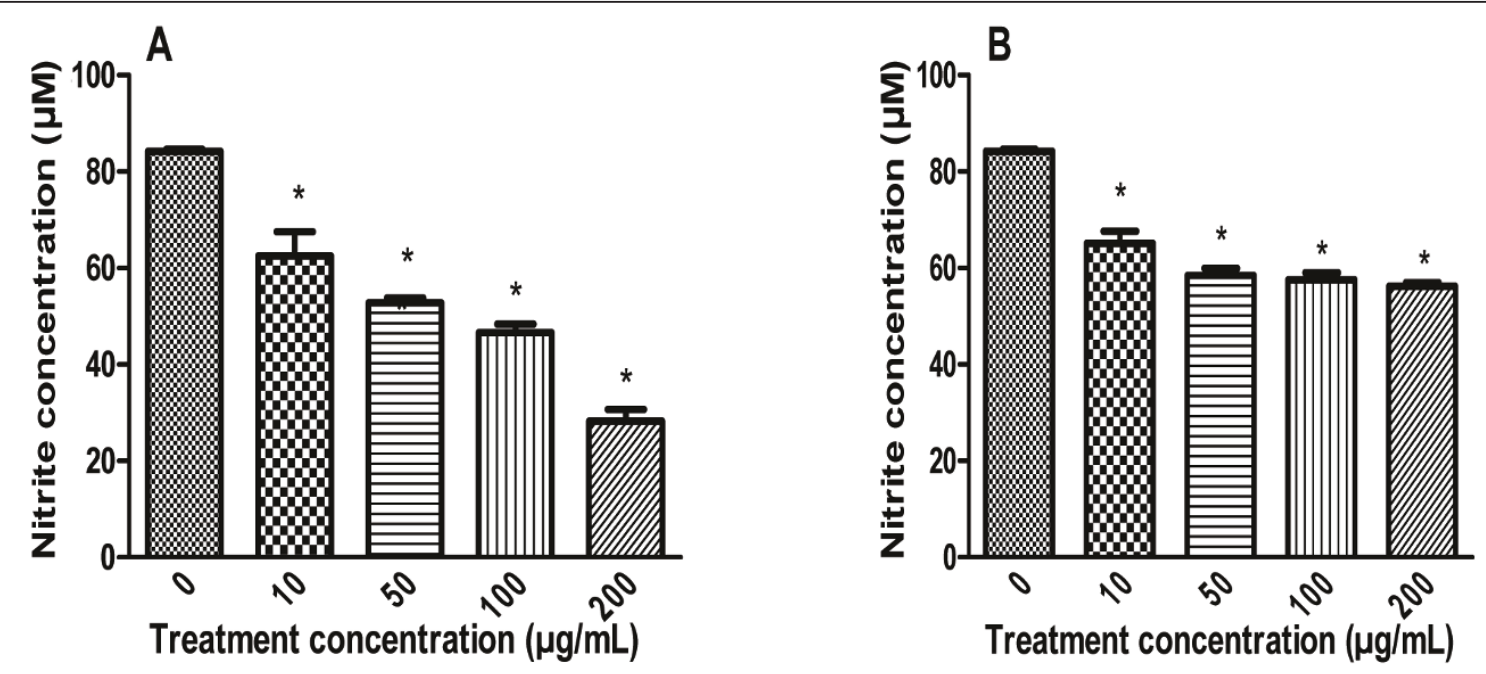

Figure 6 Effect of Fractions I and III of the ALC extract on LPS-stimulated 24 hours macrophage production of NO. Murine macrophages (RAW 264.7 cells) were pre-treated with or without the AQ and ALC extracts (10,50, 100, $200 \mu \mathrm{g} / \mathrm{ml}$ ) for two hours after which LPS $(1 \mu \mathrm{g} / \mathrm{ml})$ was added, and the NO content of the culture supernatants were determined by Griess reaction assay 24 hours later. Three independent experiments were performed and the data were shown as mean $\pm \mathrm{SD}$. Datasets were evaluated by ANOVA. ${ }^{*}$ Values $P<0.001$ compared to the LPS positive control were statistically significant.

for the management of common cold and upper respiratory infections $[9,10]$.

While ginseng is generally regarded as an immunobooster or adaptogen [1], a recent study reported that
$\mathrm{Rb}_{1}$ ginsenoside purified from an alcoholic ginseng extract induced an anti-arthritic effect in an animal model [28]. The present study also showed that the inhibitory effect of the ALC extract could be extended

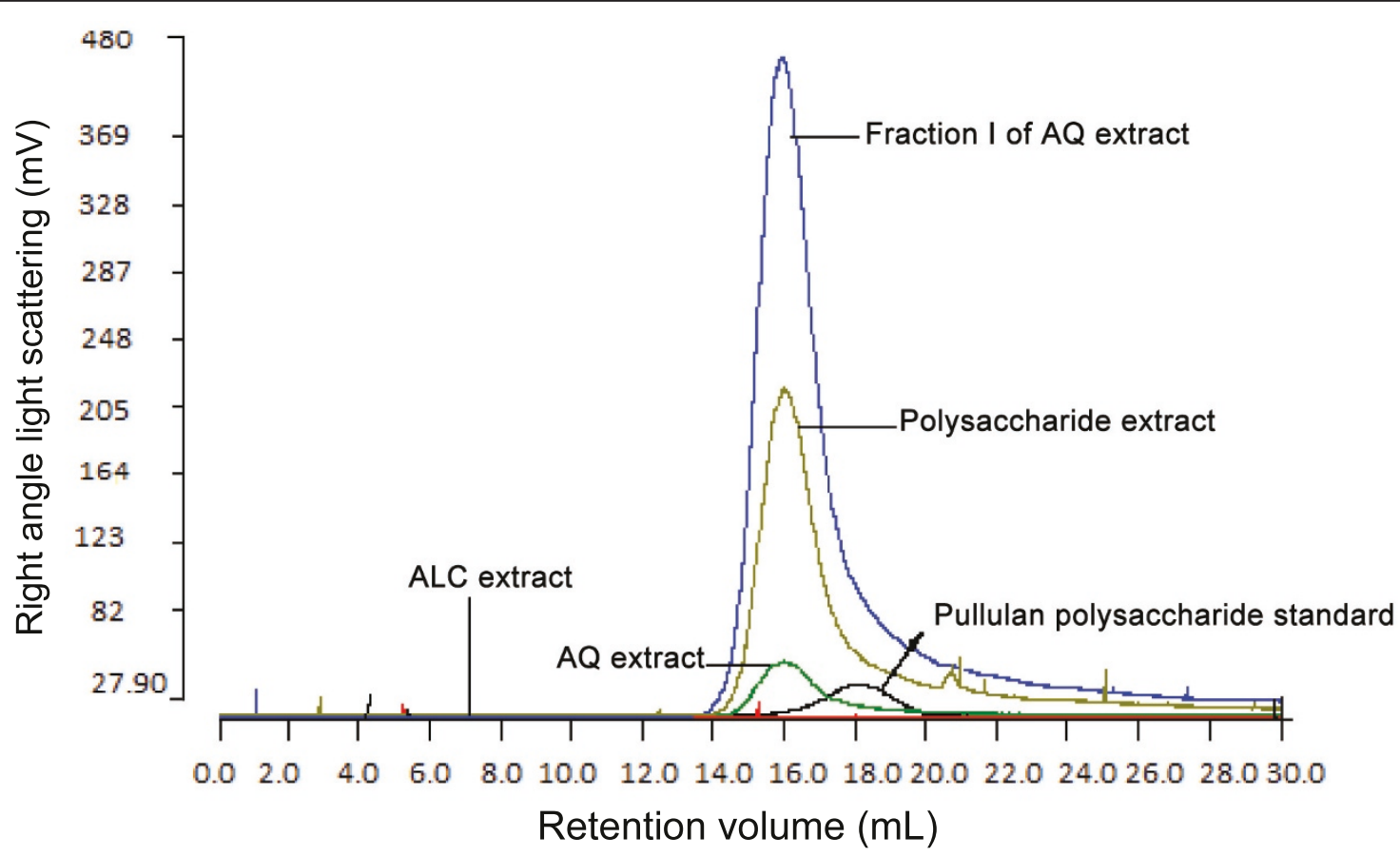

Figure 7 Identification of polysaccharides in Fraction I of the AQ extract, PS extract, AQ extract and ALC extract of ginseng by size exclusion chromatography. $100 \mu \mathrm{L}$ of $5 \mathrm{mg} / \mathrm{mL}$ sample or $2.4 \mathrm{mg} / \mathrm{mL}$ standard was injected and eluted with $0.05 \mathrm{M} \mathrm{NaNO}_{3}$ mobile phase, this was monitored with right angle light scattering detector at $1 \mathrm{~mL} / \mathrm{min}$ flow rate. Pullulan polysaccharide was used as a reference standard. The $y$ axis is the detector response $(\mathrm{mV})$ while the $x$-axis represents the retention volume $(\mathrm{mL})$. No signal was detected with the ALC extract, suggesting the absence of polysaccharides in this extract. 
to the stimulation induced by the AQ extract, suggesting that both immuno-stimulatory and immuno-suppressive components were present. Use of certain solvent systems may lead to an inactive extract. Although the magnitude of the inhibition on LPS-induced NO response by the ALC extract was quite significant, the suppressive effect was highly specific to the cytokine involved since the TNF- $\alpha$ response was not affected. Furthermore, this study showed that the ALC extract did not affect inflammatory response to LPS in monocytes and T-cells isolated from human PBMC [27]. Further studies are required to address the target cell and signalling pathways specificity of the ALC extract.

Many medicinal plants possess immuno-stimulatory activity and polysaccharides have been recognized as the primary bioactives [23]. In this study, relative abundance of PS (Fraction I) in the AQ extract (Figure 4) was well correlated with its immune-stimulatory activity (Figure 5). Plant bioactive polysaccharides were reported to have molecular weights ranging from 10,000 to $150,000 \mathrm{Da}$ $[9,10]$. The estimated molecular weight of the ginseng immuno-stimulatory PS reported in our study was within this range. Figures 1 and 5 indicated that the total macrophage-stimulating activity of the AQ extract was not solely due to Fraction I and/or the PS fraction since the immuno-stimulatory effects of the AQ extract were more potent than those of the PS or Fraction I. It is possible that some of the bioactive material was lost during isolation or fractionation procedures. It has been suggested that ginsenosides may be involved immunosuppression $[16,19,20]$, which is consistent with the higher total ginsenoside levels with the ALC extract. However, this study showed that ginsenosides $R b_{1}$ and $\operatorname{Rg}_{1}$ were not active and that the inhibitory activity was associated with macromolecular Fraction I (molecular weight of $66,000-82,000 \mathrm{Da}$ ). Figure 7 indicated that it was not PS. This finding should provide new directions for researchers exploring anti-inflammatory agents in ginseng.

Findings on the extract-specific immuno-modulatory effect have significant implications in the safety, manufacturing, production, development and regulation of products based on ginseng extracts. It is unknown whether the use of organic solvents or the extraction protocol may influence the potency and characteristics of the extracts of other ginseng species. It is imperative to carry out a systematic analysis of the physiochemical characteristics of various ginseng extracts to determine how these parameters may influence their immunomodulatory properties. The present study provides a lead for identifying immuno-bioactive constituents of ginseng.

\section{Conclusion}

ALC extract of NA ginseng, which was devoid of PS, was immuno-inhibitory whereas the AQ extract, which contained PS, was immuno-stimulatory. These extractrelated anti-inflammatory and pro-inflammatory effects may be considered as the Yin and Yang actions of ginseng.

\section{Abbreviations}

ALC: Alcoholic; AQ: Aqueous; HPLC: High Performance Liquid Chromatography; L-6: Interleukin-6; LPS: Lipopolysaccahride; NA: North America; NO: Nitric Oxide; PBMC: Peripheral Blood Mononuclear Cells; PS: Polysaccharides; TNF-a: Tumor Necrosis Factor-alpha

\section{Acknowledgements}

This research was supported by Ontario Ginseng Research \& Innovation Consortium (OGRIC) funded by the Ministry of Research \& Innovation, Ontario Research Funded Research Excellence program for the project 'New Technologies for Ginseng Agriculture and Product Development'(RE02-049 awarded to EMK Lui). We acknowledge the contribution of PolyAnalytik London Ontario, Canada in providing instrument for the gel permeation chromatography analysis of ginseng extracts.

\section{Author details}

'Ontario Ginseng Innovation and Research Consortium, the University of Western Ontario, London, Ontario, N6A 5C1, Canada. ${ }^{2}$ Department of Physiology \& Pharmacology, Schulich School of Medicine \& Dentistry, the University of Western Ontario, London, Ontario, N6A 5C1, Canada. ${ }^{3}$ Department of Chemical and Biochemical Engineering, Faculty of Engineering, the University of Western Ontario, London, Ontario, N6A 5C1, Canada.

\section{Authors' contributions}

EMKL conceived the study design, interpreted the data and wrote the manuscript. He also acquired research funding and resources. CGA carried out the experiments evaluated the immuno-modulating effects and drafted the manuscript. PAC collaborated with PolyAnalytik London Ontario, Canada to perform the gel permeation chromatography of ginseng extracts. JH performed the HPLC analysis of ginseng extracts. HP lyophilized the ginseng extracts and maintained the murine macrophages (Raw 264.7) culture. All authors read and approved the final version of the manuscript.

\section{Competing interests}

The authors declare that they have no competing interests.

Received: 1 September 2010 Accepted: 27 May 2011

Published: 27 May 2011

\section{References}

1. Angelova N, Kong HW, van der Heijden R, Yang SY, Choi YH, Kim HK, Wang M, Hankemeier T, van der Greef J, Xu G, Verpoorte R: Recent methodology in phytochemical analysis of ginseng. Phytochem Anal 2008, 19:2-16.

2. Borchers AT, Keen CL, Stern JS, Gershwin ME: Inflammation and native American medicine: the role of botanicals. Am J Clin Nutr 2000, 72:339-47.

3. Agriculture and Agri-Food Canada, Overview of the Canadian Special Crops Industry - Ginseng. [http://www.ats-sea.agr.gc.ca/can/4752-eng. htm\#ij].

4. Health Canada Drugs and Health Products, Natural Health Product Monograph Panax Ginseng. [http://www.hc-sc.gc.ca/dhp-mps/alt_formats/ pacrb-dgapcr/pdf/prodnatur/applications/licen-prod/monograph/ mono_panax_ginseng-eng.pdf].

5. Attele AS, Wu JA, Yuan CS: Ginseng pharmacology; multiple constituents and multiple actions. Biochem Pharmacol 1999, 58:1685-1693.

6. Wood PJ: The immune system: recognition of infectious agents. Anaesth Intensive Care Med 2006, 7:179-180. 
7. Bondeson J: The mechanisms of action of disease-modifying antirheumatic drugs: a review with emphasis on macrophage signal transduction and the induction of proinflammatory cytokines. Gen Pharmacol 1997, 29:127-150.

8. Lee MY, Park BY, Kwon OK, Yuk JE, Oh SR, Kim HS, Lee HK, Ahn KS: Antiinflammatory activity of (-)-aptosimon isolated from Daphne genkwa in RAW264.7. Int Immunopharmacol 2009, 9:878-885.

9. Biondo PD, Goruk S, Ruth MR, O'Connell E, Field CJ: Effect of CVT-E002 ${ }^{\text {TM }}$ (COLD-fX ${ }^{\circledR}$ ) versus a ginsenoside extract on systemic and gut-associated immune function. Int Immunopharmacol 2008, 8:1134-1142.

10. Wang M, Guilbert LJ, Li J, Wu Y, Pang P, Basu TK, Shan JJ: A proprietary extract from North American ginseng (Panax quinquefolium) enhances IL-2 and IFN-gamma productions in murine spleen cells induced by Con-A. Int Immunopharmacol 2004, 4:311-315.

11. Jie $\mathrm{YH}$, Cammisuli S, Baggiolini M: Immunomodulatory effects of Panax Ginseng C.A. Meyer in the mouse. Agents Actions 1984, 15:3-4

12. Friedl R, Moeslinger T, Kopp B, Spieckermann PG: Stimulation of nitric oxide synthesis by the aqueous extract of Panax ginseng root in RAW 264.7 cells. Br J Pharmacol 2001, 134:1663-1670.

13. Assinewe VA, Arnason JT, Aubry A, Mullin J, Lemaire I: Extractable polysaccharides of Panax quinquefolius L. (North American ginseng) root stimulate TNF-a production by alveolar macrophages. Phytomedicine 2002, 9:398-404.

14. Lee JW, Takano-Ishikawa Y, Watanabe J, Kobori M, Tsushida T, Yamaki K: Effect of ginsenosides and red ginseng water extract on tumor necrosis factor-a production by rat peritoneal macrophages. Food Sci Technol Res 2002, 8:300-303.

15. Zhou DL, Kitts DD: Peripheral blood mononuclear cell production of TNF$a$ in response to North American ginseng stimulation. Can J Physiol Pharmacol 2002, 80:1030-1033.

16. Lee DC, Yang CL, Chik SC, Li JC, Rong JH, Chan GC, Lau AS: Bioactivityguided identification and cell signalling technology to delineate the immunomodulatory effects of Panax ginseng on human promonocytic U937 cells. J Trans/ Med 2009, 34:1-10.

17. Park JS, Park EM, Kim DH, Jung K, Jung JS, Lee EJ, Hyun JW, Kang JL, Kim HS: Anti-inflammatory mechanism of ginseng saponins in activated microglia. J Neuroimmunol 2009, 209:40-49.

18. Li J, Ichikawa T, Nagarkatti $P$, Nagarkatti M, Hofseth LJ, Windust A, Cui T: American ginseng preferentially suppresses STAT/iNOS signaling in activated macrophages. J Ethnopharmacol 2009, 125:145-150.

19. Rhule A, Rase B, Smith JR, Shepherd DM: Toll-like receptor ligand-induced activation of murine DC2.4 cells is attenuated by Panax notoginseng. J Ethnopharmacol 2008, 28:179-186.

20. Rhule A, Navarro S, Smith JR, Shepherd DM: Panax notoginseng attenuates LPS-induced pro-inflammatory mediators in RAW264.7 cells. Ethnopharmacol 2006, 106:121-128.

21. Liou CJ, Huang WC, Tseng J: Long-term oral administration of ginseng extract modulates humoral immune response and spleen cell functions. Am J Chin Med 2005, 33:651-661.

22. Jin UH, Park SG, Suh SJ, Kim JK, Kim DS, Moon SK, Lee YC, Park WH, Kim CH: Inhibitory effect of Panax notoginseng on nitric oxide synthase, Cyclo-oxygenase-2 and neutrophil functions. Phytother Res 2007, 21:142-148.

23. Schepetkin IA, Quinn MT: Botanical polysaccharides: macrophage immunomodulation and therapeutic potential. Int Immunopharmacol 2006, 6:317-333.

24. Yue PY, Mak NK, Cheng YK, Leung KW, Ng TB, Fan DT, Yeung HW, Wong RN: Pharmacogenomics and the Yin/Yang actions of ginseng: antitumor, angiomodulating and steroid-like activities of ginsenosides. Chin Med 2007, 2:1-21.

25. lishi $H$, Tatsuta $M$, Baba M, Uehara $H$, Nakaizumi A, Shinkai $K$, Akedo $H$ Funai H, Ishiguro S, Kitagawa I: Inhibition by ginsenoside Rg3 of bombesin-enhanced peritoneal metastasis of intestinal adenocarcinomas induced by azoxymethane in wistar rats. Clin Exp Metastasis 1997, 15:603-611.

26. Tatsuka M, Maeda M, Ota T: Anticarcinogenic effect and enhancement of metastatic potential of BALB/c 3T3 cells by ginsenoside Rh (2). Jpn J Cancer Res 2001, 92:1184-1189.

27. Toth JM, Hewson GL, Frodermann V, Chau LA, Azike C, Lui E, Madrenas J: Modulatory effects of ginseng extracts on human innate and adaptive immune responses. Proceedings of the 10th International Symposium on Ginseng: 13-16 September, 2010; Seoul, Korea

28. Kim HA, Kim S, Chang SH, Hwang HJ, Choi YN: Anti-arthritic effect of ginsenoside $\mathrm{Rb}_{1}$ on collagen induced arthritis in mice. Int Immunopharmacol 2007, 7:1286-1291.

doi:10.1186/1749-8546-6-21

Cite this article as: Azike et al:: The Yin and Yang actions of North American ginseng root in modulating the immune function of macrophages. Chinese Medicine 2011 6:21.

\section{Submit your next manuscript to BioMed Central and take full advantage of:}

- Convenient online submission

- Thorough peer review

- No space constraints or color figure charges

- Immediate publication on acceptance

- Inclusion in PubMed, CAS, Scopus and Google Scholar

- Research which is freely available for redistribution

Submit your manuscript at www.biomedcentral.com/submit
Ciomed Central 